\title{
A fuzzy ELECTRE structure methodology to assess big data maturity in healthcare SMEs
}

\author{
Alejandro Peña · Isis Bonet - Christian Lochmuller • Marta S. Tabares · Carlos C. \\ Piedrahita - Carmen C. Sánchez · Liliana M. Giraldo · Mario Góngora · Francisco \\ Chiclana
}

Received: date / Accepted: date

\begin{abstract}
Advances in technology and an increase in the amount and complexity of data that is generated in healthcare have led to an indispensable revolution in this sector related to big data. Analytics of information based on multimodal clinical data sources require big data projects. When starting big data projects in the healthcare sector, it is often necessary to assess the maturity of an organization with respect to big data, i.e. its capacity in managing big data. The assessment of the maturity of an organization requires multi criteria decision making as there is no single criterion or dimension that defines the maturity level regarding big data but an entire set of them. Based on the ISO 15504, this article proposes a fuzzy ELECTRE structure methodology to assess the maturity level of small and medium-sized enterprises in the healthcare sector (SMEHs). The obtained experimental results provide evidence that this methodology helps to determine and compare maturity levels in big data management of organizations or the evolution of maturity over time. This is also useful in terms of diagnosing the
\end{abstract}

Alejandro Peña, Isis Bonet, Christian Lochmuller

University EIA, Envigado, Colombia; E-mail: fjapena@eia.edu.co, isis.bonet@eia.edu.co, christian.lochmuller@eia.edu.co

Marta S. Tabares

Universidad EAFIT, Medellín, Colombia; E-mail: mtabares@eafit.edu.co

Carlos C. Piedrahita, Carmen C. Sánchez, Liliana M. Giraldo

Universidad de Medellín, Colombia; E-mail: cpiedrahita@udem.edu.co,_ccsanchez@udem.edu.co,_lmgiraldo@udem.edu.co

\section{Mario Góngora}

Institute of Artificial Intelligence, De Montfort University, Leicester, UK; E-mail: mgongora@dmu.ac.uk

Francisco Chiclana

Dept. of Computer Science and Artificial Intelligence, University of Granada, Spain; and Institute of Artificial Intelligence, De Montfort University, Leicester, UK; E-mail: inv.chiclana@ugr.es; chiclana@dmu.ac.uk readiness of an organization before starting to implement big data initiatives or technologies.

Keywords Big data Healthcare - Maturity level · ELECTRE method · Fuzzy methods · Outranking

\section{Introduction}

Advances in technology during the last decade have led to an exponential growth in data generation and storage. Data comes for example from different technological devices that collect information about what we do. Accordingly, technology for data analysis and the understanding of information is evolving. With these developments, the concept of big data has emerged $[1,2]$. This concept is already helping industries to become more efficient and effective [3], including organizations in the health care sector. In general, "big data" not only refers to large volumes of data that organizations manage, but also to the wide variety of data sources and types. Additionally, this concept refers to the velocity with which data is generated or changed. These three characteristics of a basic definition of the term big data are known as the 3 V's of big data $[4,5]$. The HealthCare industry has also entered the big data realm, because research, innovation, product and service development require big data management. This goes beyond improving benefits and reducing costs in this industry. Big data is used to predict epidemics, cure diseases, improve the quality of patients life as many decisions in these fields are directed by evidence and facts [4, 6-8].

Due to the wide variety of specialties that characterize the HealthCare sector, different types of organizations exist. Many of these organizations are small and medium-sized enterprises (SMEHs). So it is understandable that the implementation and adoption of information technologies centered on big data varies widely [7]. In order to be competi- 
tive in a globalized market, SMEHs have carried out a series of actions tending to implement information systems that improve their market possibilities in terms of innovation, agility and efficiency [9]. This is not always an easy task, as in many cases the required investment, personnel for data processing or the need and difficulty to find a tailored IT solution are challenging $[10,11]$. Consequently, the conditions for starting a big data initiative or project vary between organizations or parts of the same organization. That is why organizations, including SMEs and SMEHs, need to assess their level of maturity or how capable they are in managing big data processes. This assessment is done to establish a baseline or benchmark, which refers to comparing an organization to competitors, market leaders or best practice.

To assess the maturity level of SMEs, and of SMEHs in particular, four well-defined development trends can be identified. A first trend focuses on the development of models, mainly based on qualitative information, as support for decision making under uncertainty in organizations [13-16]. In this area a first work can be highlighted, in which the guidelines for the selection of investment projects are established, using for this purpose a fuzzy model, which is integrated with the ELECTRE methodology [12]. The work presents a methodology that allows the modeling of different decision criteria by using the concepts of fuzzy logic. In this same line of research multicriteria ELECTRE models can be found that incorporate sub criteria for the evaluation of logistics platforms that improve distribution channels of organizations [17]. Another study outlines a pair of models that help decision making under uncertainty using different criteria, such as: ER - Evidential Reasoning and AHP - Analytical Hierarchy Process, for the localization of healthcare infrastructure, taking into account the optimal point of operation of an organization [18]. One of the most promising areas for the application of multicriteria models are the SMEs (Small and Medium-sized Enterprises), where ELECTRE has shown its potential in allocating loans to start-up companies, where a series of qualitative criteria are used, which characterize the start-up and its market operations [19]. The use of qualitative information in ELECTRE models is a key element of these models. A modified ELECTRE model has been proposed for the acquisition of agricultural products through the use of mobile devices, eliminating the subjectivity associated with the buying decision and this type of products [20].

A second development trend is centered on the analysis of the characteristics, the questioning and challenges related to the management of big data in organizations. In a first article, different free tools (OSS - Open Source Software) for carrying out data analysis have been analyzed. In this work the main characteristics (ownership; workload; source code; low latency; complexity) that these tools must possess according to the qualities of big data in organizations in differ- ent sectors of the economy are described [21]. The research literature provides evidence for the growing need of SMEs to work with big data, as they now handle high volumes of information, finally with the aim to achieve a closer relationship with their clients, or patients in the health sector. As an alternative answer to the challenge of big data management, cloud computing solutions are a trend. However, SMEs have been "reluctant" to use tools on the Internet, sometimes due to some technical limitations, as in many regions of the world SMEs still do not have access to the internet [4]. In this line of research, the benefits of applying free tools for big data in SMEs are evident, because this type of organizations often does not have sufficient financial, human and technological resources to put into operation, contract, maintain or outsource a technological infrastructure based on commercial big data tools and services.

A third trend focuses on the maturity models used in organizations for software development, where a first article emphasizes the importance of maturity models in software engineering in small and medium-sized enterprises (MISSMEs) [22]. Thus, many companies are implementing processes for the improvement of models, seeking to increase the maturity of software development processes, where the adaptation and application of ISO/IEC 15504, a reference for maturity models, has allowed to decrease the amount of resources required by SMEs for software development in Spain [23]. In [11] a comprehensive bibliographical review is presented that seeks to consolidate a list of maturity evaluation models, associated with the challenges, benefits and results that these can bring to organizations $[11,24]$. Accordingly, many of the maturity models have to do with improving and optimizing the software development process and with increasing the flexibility and adaptability of the software to the business processes of an organization [25]. Additionally, free software (OSS - Open Source Software) is a paradigm for software development, in which groups are used for the collaborative development, so pooling knowledge and skills can lead to a better quality of the products, compared to conventional software development methods. However, literature is not always clear in the use of methods for the evaluation of OSS, this is why a maturity evaluation method for OSS has been proposed by the developer community [26].

A fourth development trend focuses on the use of analytical tools to assess the maturity level of big data processes in organizations, where in [27] a frame of reference for the capture, modeling and analysis of the evolution of software models worldwide is established. For this analysis, a series of analytical indicators of maturity are defined for organizations, based on the DELTA model, which evaluates five aspects of the organization. These aspects are the following [28]: (1) Data, (2) enterprise or organization, (3) leadership, (4) targets or techniques and applications, and (5) the ana- 
lysts who apply these techniques. The set of these aspects shows at what stage or level of maturity the organizations is positioned, in terms of the use of analytical techniques for data processing. In a later article it is shown how projects can be developed for the measurement of maturity, gathering the most important characteristics used for the development of maturity models $[29,30]$. Also, and independently of a specific business process, there is an article that mentions how to incorporate the concepts of maturity into Business Process Management (BPM), considering specific BPM aspects for each organization [31]. A recent article that presents a systematic review of the BPMMs (Business Process Maturity Model) most commonly used by the organizations, reveals that although many of the BPMMs have been developed in the last decade, these still have an incipient use and are showing that empirical studies must be carried out in order to demonstrate the usefulness of the implementation of BPMMs, separating the evaluation method from the maturity model as such, which acts as a frame of reference for the evaluation of the performance of the processes [32].

Despite this development with respect to maturity models and the exponential growth of data that SMEs worldwide have experienced, there is a lack of models that allow to measure the level of maturity of software and the use of big data tools, that are commercial or of the OSS type, to support organizations BPM processes. In particular, there is a need for models that evaluate the maturity level by using tools that integrate highly qualitative information and lead to decisions under uncertainty with regard to the acquisition, improvement and adaptation of technology, according to the requirements that emerged with big data in SMEs in the HealthCare industry.

In this article a multicriteria fuzzy model is proposed to evaluate the degree of maturity of big data management in organizations - in particular, in organizations that operate in the health sector. The proposed model allows to evaluate how mature or capable an organization or parts of it are with respect to the management of big data processes. This is done with the main objective of creating the conditions to establish a baseline in an organization. This will reveal, if the organization or a part of it, is mature enough to initiate big data initiatives or projects, or, in the opposite case, to indicate the aspects that must be improved before reaching a level of maturity that allows to undertake these projects with a greater probability of success. However, determining the maturity level with respect to big data is also useful for tracking the evolution of maturity in an organization over time or comparing it to the competition or market leaders. For the development of the here proposed model, five dimensions of the business are considered and evaluated. According to a 'Transforming Data with Intelligence' (TDWI) big data methodology (https://tdwi.org/), these are [33]:

- Infrastructure,
- Data management,

- Organization,

- Governance and

- Analysis.

Where each of the dimensions is defined by a series of characteristics. These will be described in a qualitative way, according to the principles of fuzzy logic. The degree of maturity will be evaluated in terms of the criteria defined by a set of decision makers matching the before mentioned dimensions and applying the structure of a Fuzzy ELECTRE rating model [12]. A decision maker is an expert who provides the input to the model, based on his knowledge and experience. The degree of maturity obtained from the proposed model will allow SMEHs to take a series of actions. These aim at the optimization, improvement and acquisition of information technologies and of big data that allow them the generation of competitive market advantages derived from the management of large amounts of information from different sources [34].

The following section presents the methodology that has been applied for the development of the proposed model. The methodology allows characterizing the maturity of big data processes, the definition of the dimensions as well as their associated criteria. The here applied methodology is based on the TDWI methodology. In this context, the structure of the rating model, as well as the definition of decision makers in terms of the criteria that define each dimension will be described. In this same section a basic case study is presented to describe the operation of the model. Subsequently, the analysis of experimental results is outlined in a separate section, which focuses mainly on the analysis of the model in terms of different hypothetical SMEHs, based on the criteria defined for each dimension. This leads to the main conclusions and recommendations of this work that allow organizations to address big data challenges within SMEHs in the HealthCare sector, from the perspective of measuring the level of maturity in big data management.

\section{Methodology}

One of the starting points for assessing the level of maturity in big data management of a SMEHs is the characterization of a series of criteria regarding the operations of the business. The evaluation of maturity is done through the solution of a multicriteria problem with qualitative information, for which a methodology is proposed that consists of the following stages [19]: Definition of the evaluation criteria, definition of the fuzzy sets that establish the levels of maturity and definition of the importance of each of the criteria used in the maturity evaluation. 


\subsection{Selection of the evaluation criteria}

The methodology TDWI [33] was used to select the criteria for assessing the maturity of big data management in SMEHs, which allows a deeper understanding of the business and the technical challenges associated with the use of tools for business intelligence (BI) and data warehousing (DW). Accordingly, the TDWI methodology establishes five stages of maturity: nascent, pre-adoption, early adoption, corporate adoption and mature / visionary. These stages imply that an organization, which operates for example at the "early adoption" stage, has to go through the stage of "corporate adoption" in order to get to a higher level of maturity. Where "corporate adaption" requires the measurement of maturity for the entire organization, and not only parts of it. That means that the scope of measurement and maturity broadens and refers to the corporate level. A company must pass through this level to reach, if this is the objective of an organization, the highest level, which is named "mature / visionary." Consequently, the achievement of maturity levels follows a stage gate process. In this sense, for the organization the stages also represent, in a prescriptive way, a route of development to follow.

For the case of the proposed model, and according to the characteristics that define a SMEH, the definition of the TDWI and the maturity assessment outlined in [35], the following five levels of big data maturity are applied:

\section{"Inexistent" $>$ "Exploring" $〉$ "Analyzing" $>$ "Integrating" $>$ "Innovating"}

Fig. 1 Measurement scale for big data maturity management

Level 0 - "Inexistent". The organization, due to the volume of data it handles, does not treat or process big data at present. Consequently, big data management is inexistent. The organization is "immature" with respect to the management of big data (level 0 ).

Level 1 - "Exploring". The organization is exploring the topic of big data and / or is planning to start a big data initiative.

Level 2 - "Analyzing". The organization is getting involved in basic data analysis (big data) and seems dedicated to take the step towards defining and communicating its "big data strategy".

Level 3 - "Integrating". The organization is already integrating massive data from traditional data sources and business processes to gain knowledge and an integrated understanding of them.

Level 4 - "Innovating". The organization is fully capable of running big data projects successfully. Big data is being treated as an asset of the organization and this asset is used to innovate and create new products and services.
These levels, which will be applied for the proposed model, are similar to the levels of other maturity models, e.g. to the ISO 15504 standard, which defines the following levels: Level 0 - Immature organization, Level 1 - Basic organization, Level 2 - Managed Organization, Level 3 - Established Organization, Level 4 - Predictable Organization, Level 5 Optimized Organization.

Table 1 enumerates, at the level of an example, the building blocks for a big data maturity evaluation. These include dimensions, criteria and characteristics for a maturity evaluation.

Table 1 Categories of Dimensions, Criteria and Characteristics (example)

\begin{tabular}{|c|c|c|}
\hline Dimension & Criteria & Characteristics \\
\hline \multirow{2}{*}{$G^{1}$ : People } & $\mathbf{g}_{1}^{1}$ : Staffing/Training & 5 \\
\hline & $\begin{array}{l}\mathbf{g}_{2}^{1}: \text { Business Fo- } \\
\text { cus/Commitment }\end{array}$ & 5 \\
\hline \multirow[t]{3}{*}{$G^{2}$ : Process } & $\begin{array}{l}\mathbf{g}_{\mathbf{1}}^{\mathbf{2}} \text { : Big Data Man- } \\
\text { agement \& Gover- } \\
\text { nance }\end{array}$ & 8 \\
\hline & $\mathbf{g}_{2}^{2}:$ Policy & 3 \\
\hline & $\begin{array}{l}\mathbf{g}_{3}^{2}: \text { Business Fo- } \\
\text { cus/Commitment }\end{array}$ & 5 \\
\hline$G^{3}:$ Technology & $\begin{array}{l}\mathbf{g}_{1}^{\mathbf{3}} \text { : Technology in } \\
\text { Place }\end{array}$ & 4 \\
\hline \multirow{3}{*}{$G^{4}:$ Data } & $\mathbf{g}_{1}^{4}$ : Data Volume & 3 \\
\hline & $\mathbf{g}_{2}^{\mathbf{4}}:$ Data Type & 6 \\
\hline & $\mathbf{g}_{3}^{\mathbf{4}}$ : Data Quality & 5 \\
\hline
\end{tabular}

\subsubsection{Definition of the dimensions used in the evaluation}

For the evaluation of big data management maturity in a SMEHs four dimensions have been proposed [28]:

\section{$G^{1}$ : Human resources and talent.}

- People, their skills, abilities and leadership in big data.

- The strategic approach and the commitment of the management with respect to big data.

\section{$\mathrm{G}^{2}$ : The processes.}

- Big data management and big data governance.

- Policies for big data.

- Business objectives and big data.

$G^{3}$ : The technology.

- Available technology for big data.

$G^{4}$ : The data. 
- The volume of big data.

- Data types and sources.

- Data quality.

\subsubsection{Definition of criteria and characteristics associated with the dimensions of big data maturity}

Based on [35] the following 44 criteria are defined for the dimensions, which determine the maturity of big data management in an organization.

g1 $_{1}^{1}$ : The people, their skills, abilities and their leadership in big data.

$\mathbf{g}_{11}^{1}$ : The organization, i.e. the SMEH, has the staff, the skills, capacities and time that is required to execute a large data project, either with internal staff, through new hires or outsourcing.

$\mathbf{g}_{12}^{1}$ : There is a budget for training and instructing people to manage big data.

$\mathbf{g}_{13}^{1}$ : Sufficient collaborators are available to implement, support and maintain any new initiative or implementation regarding big data.

$\mathbf{g}_{\mathbf{1 4}}^{\mathbf{1}}$ : Staff is allocated to implement, support and maintain any new initiative or implementation with respect to corporate analytics regarding big data.

$\mathbf{g}_{15}^{\mathbf{1}}$ : The organization is willing to bring in external advisors or consultants for issues, where internal staff does not have the required skills.

$g_{2}^{1}$ : The strategic approach and commitment of the management in big data.

$\mathbf{g}_{\mathbf{2 1}}^{\mathbf{1}}$ : Planning for big data initiatives is a collaborative effort to meet the objectives of the organization, defined by top management and implemented by the ICT department.

$\mathbf{g}_{\mathbf{2} 2}^{\mathbf{1}}$ : The organization believes in sharing the knowledge, comprehension or understanding obtained from big data among the business units of the organization.

$\mathbf{g}_{\mathbf{2 3}} \mathbf{1}$ : Top management has a vision for the future of big data in the organization.

$\mathbf{g}_{\mathbf{2 4}}^{\mathbf{1}}$ : Top management considers big data as one of the key elements for meeting future business goals.

$\mathbf{g}_{\mathbf{2 5}}^{\mathbf{1}}$ : The different business units are willing to adopt big data as a new standard within the organization.

$\mathrm{g}_{1}^{2}$ : Big data management and big data governance.

$\mathbf{g}_{\mathbf{1 1}}^{2}$ : The organization has hired full-time employees, in ICT or business areas, to assume specific roles and functions in big data.

$\mathbf{g}_{12}^{2}$ : The organization has full-time employees dedicated to the analysis of big data, either ICT or business areas.

$\mathbf{g}_{\mathbf{1 3}}^{\mathbf{2}}$ : A steering committee (or equivalent) is in place to direct the big data approach.

$\mathbf{g}_{\mathbf{1 4}}^{\mathbf{2}}$ : Well-defined processes to digitize data are implemented throughout the organization. $\mathbf{g}_{15}^{2}$ : Access to a big data "platform" or "system" is provided through a well-defined process.

$\mathbf{g}_{\mathbf{1 6}}^{\mathbf{2}}$ : The organization knows and has a clear understanding, what types of data are currently used and available.

$\mathbf{g}_{17}^{2}$ : Access to data sources can be provided in an agile and timely manner, when necessary.

$\mathbf{g}_{\mathbf{1 8}}^{2}$ : Big data is managed as an integral part of the business architecture of the organization.

$\mathrm{g}_{2}^{2}$ : Policies regarding big data.

$\mathbf{g}_{\mathbf{2 1}}^{2}$ : The organization complies with the laws and regulations (privacy legislation, like "habeas data" in Colombia, etc.).

$\mathbf{g}_{\mathbf{2} 2}^{2}$ : The data retention policy is aligned with the strategies of making data available for decision-making, knowledge-seeking and analysis.

$\mathbf{g}_{\mathbf{2 3}}^{\mathbf{2}}$ : Security policies to protect data storage and transmission are established.

$\mathbf{g}_{3}^{2}$ : Business objectives and big data.

$\mathbf{g}_{\mathbf{3 1}}^{2}$ : A big data program for the organization is in place, which is closely aligned with business areas and objectives.

$\mathbf{g}_{\mathbf{3 2}}^{\mathbf{2}}$ : Quantifiable metrics are applied to assess the success of big data activities or initiatives.

$\mathbf{g}_{\mathbf{3 3}}^{\mathbf{2}}$ : The big data program or activities of the organization have a dedicated and revolving budget for infrastructure and support.

$\mathbf{g}_{\mathbf{3 4}}^{\mathbf{2}}$ : The organization is applying big data analytics, clearly defined tools and methods.

$\mathbf{g}_{\mathbf{3 5}}^{\mathbf{2}}$ : The organization is trying to incorporate big data as a component of its projects or programs, daily work and business processes.

$\mathrm{g}_{1}^{3}$ : Technology available for big data.

$\mathbf{g}_{11}^{\mathbf{3}}$ : The organization is willing to use new platforms like Hadoop to store and process massive data.

$\mathbf{g}_{12}^{\mathbf{3}}$ : Currently, the organization is analyzing available data and generating reports or scorecards from that data.

$\mathbf{g}_{\mathbf{1 3}}^{\mathbf{3}}$ : The organization can secure and encrypt large amounts of data.

$\mathbf{g}_{\mathbf{1 4}}^{\mathbf{3}}$ : At present, the organization has the necessary technological infrastructure to integrate data from different sources, structures, types, and platforms.

$\mathrm{g}_{1}^{4}$ : The volume of big data.

$\mathbf{g}_{\mathbf{1 1}}^{\mathbf{4}}$ : The organization administrates a volume of data which is less than 1 Terabyte (TB).

$\mathbf{g}_{12}^{\mathbf{4}}$ : The organization administrates a volume of data that is between 1 Terabyte (TB) and 1 Petabyte (PB).

$\mathbf{g}_{\mathbf{1 3}}^{\mathbf{4}}$ : The organization administrates a volume of data which is bigger than 1 Petabyte (PB).

$\mathrm{g}_{2}^{4}$ : Data sources and types. 
$\mathbf{g}_{21}^{4}$ : Currently, the organization is already taking advantage of structured data (transaction data, payroll data, etc.).

$\mathbf{g}_{22}^{4}$ : The organization is capitalizing on unstructured data (blogs, video, social media, documents).

$\mathbf{g}_{\mathbf{2 3}}^{\mathbf{4}}$ : The organization is exploiting more than $90 \%$ of its internal data (which is being leveraged).

$\mathbf{g}_{24}^{4}$ : The organization is trying to take advantage of external data.

$\mathbf{g}_{\mathbf{2 5}}^{\mathbf{4}}$ : The organization is integrating data from internal and external sources for big data projects.

$\mathbf{g}_{\mathbf{2 6}}^{\mathbf{4}}$ : The organization integrates the knowledge, comprehension or understanding (insights) obtained from big data in its processes and decision making.

$\mathrm{g}_{3}$ : The quality of the data.

$\mathbf{g}_{\mathbf{3 1}}^{\mathbf{4}}$ : The organization?s data and meta data are of high quality, complete and consolidated.

$\mathbf{g}_{\mathbf{3 2}}^{\mathbf{4}}$ : End users in the organization rely on the quality of data.

$\mathbf{g}_{\mathbf{3 3}}^{\mathbf{4}}$ : The organization converts data, so that the result is "consumable" (ready to be processed by the "end user").

$\mathbf{g}_{\mathbf{3 4}}^{\mathbf{4}}$ : The organization has a well-established data hierarchy (for example, concerning the relationship of a category in healthcare or medicine with sub-categories or areas)).

$\mathbf{g}_{\mathbf{3 5}}^{\mathbf{4}}$ : The organization is applying some international quality standard for data management.

\subsection{Importance of each of the evaluation criteria}

One of the most important difficulties in adopting a model of multiple criteria decision aid or analysis (MCDA) is to determine the importance of each of the criteria that represent the model. For this purpose, different methods have been proposed in literature to determine this importance [36-43].

The concept of "criteria" has different definitions according to the compensatoriness of the multicriteria method to be used [44], where the concept of compensatoriness defines the nature and degree of trade-offs between the criteria. In a completely compensatory methodology, such as the Multi-Attribute Utility Theory [45], weights are interpreted as trade-offs, and these depend on the scale and range of the criterion. In non-compensatory models, such as ELECTRE [46] and PROMETHEE [47], the weights take an intrinsic value according to the measure of importance of a criterion and is independent of the scale used.

Each of the characteristics associated with each criterion is evaluated by a decision maker (DM), considering the following qualitative numerical scale:

1. Do not know,

2. Disagree,
Table 2 Range of qualities that describe the average of the grades associated with each of the dimensions

\begin{tabular}{llc}
\hline LTs (linguistic term) & Average $(p)$ & Scale $\left(x c_{i}\right)$ \\
\hline Extremely Good (EG) & $p=5$ & 9 \\
Very Good (VG) & $p<5$ & 7 \\
Good (G) & $p<4$ & 5 \\
Medium Bad (MB) & $p<3$ & 3 \\
Bad (B) & $p<2$ & 2 \\
Very Bad (VB) & $p=1$ & 1 \\
\hline
\end{tabular}

3. Neither disagree nor agree,

4. Agree,

5. Strongly agree.

In this way, the value reached by each criterion can be calculated as the average of the score for each of the characteristics that are grouped in Table 2 [12].

According to this numerical qualitative scale, each dimension can be represented as a linguistic variable, where the fuzzy sets can be defined as follows [12]:

$u_{j s, i}=\exp \left(-\frac{1}{2}\left(\frac{\left.x c_{j s, i}-G^{i}\right)}{\sigma_{d}}\right)^{2}\right)$

where $i$ represents the number of dimensions or characteristics that allow to evaluate the level of maturity $(i=1,2, \ldots, n c)$; $j s$ indicates the number of fuzzy sets for the linguistic scale variable according to Table $2(j s=1,2, \ldots, 6) ; n c$ indicates the number of criteria; and $u_{j s, i}$ indicates the membership value for a dimension of belonging to a fuzzy set that constitutes the linguistic scale variable.

According to $[12,48]$ and equation (1), which defines the structure of the fuzzy sets associated with each criterion, the numeric definition is as follows:

Table 3 The 1-9 Fuzzy conversion scale - Gaussian Fuzzy Sets

\begin{tabular}{ccc}
\hline LTs & Centroids & $\sigma$ \\
\hline 1 & 1 & 0 \\
2 & 2 & 0.4 \\
3 & 3 & 0.6 \\
5 & 5 & 1.0 \\
7 & 7 & 1.4 \\
9 & 9 & 1.8 \\
\hline
\end{tabular}

Table 3 shows that the fuzzy sets increase their base as maturity levels increase. That is due to the fact that a wider 
scale is required to explain higher levels of maturity for different dimensions, seeking to achieve greater differentiation with respect to the criteria of "organization", "infrastructure" and "big data management strategy" [33].

\subsection{Experimental Design}

For the analysis and validation of the proposed model, the qualitative decision matrix will be constructed. This is done through the participation of four decision makers (DM), who are experts in this area of knowledge. For this matrix five levels of maturity (inexistent, exploring, analyzing, integrating, innovating) are used as the DM is considering each of the criteria for each group of dimensions to evaluate big data maturity based on this structure. The evaluation is done by using the LTs (linguistic terms) shown in Table 2.

Table 4 Example: Fuzzy decision matrix with five defined maturity levels for application in a SMEH

\begin{tabular}{|c|c|c|c|c|c|}
\hline Maturity level & Criterion & DM1 & DM2 & DM3 & DM4 \\
\hline \multirow{4}{*}{ Inexistent (P0) } & $\mathrm{C} 1$ & VB & VB & MB & B \\
\hline & $\mathrm{C} 2$ & B & VB & MB & MB \\
\hline & $\mathrm{C} 3$ & VB & VB & B & B \\
\hline & $\mathrm{C} 4$ & B & VB & MB & MB \\
\hline \multirow{4}{*}{ Exploring (P1) } & $\mathrm{C} 1$ & $\mathrm{G}$ & VG & VG & G \\
\hline & $\mathrm{C} 2$ & $\mathrm{G}$ & VG & MB & MB \\
\hline & $\mathrm{C} 3$ & VG & $\mathrm{G}$ & G & G \\
\hline & $\mathrm{C} 4$ & G & G & MB & MB \\
\hline \multirow{4}{*}{ Analyzing (P2) } & $\mathrm{C} 1$ & G & G & G & G \\
\hline & $\mathrm{C} 2$ & MB & $\mathrm{G}$ & MB & MB \\
\hline & $\mathrm{C} 3$ & B & MB & MB & B \\
\hline & $\mathrm{C} 4$ & $\mathrm{G}$ & MB & B & MB \\
\hline \multirow{4}{*}{ Integrating (P3) } & $\mathrm{C} 1$ & MB & VG & MB & MB \\
\hline & $\mathrm{C} 2$ & $\mathrm{MB}$ & $\mathrm{MB}$ & B & B \\
\hline & $\mathrm{C} 3$ & G & $\mathrm{MB}$ & MB & B \\
\hline & $\mathrm{C} 4$ & MB & MB & $\mathrm{B}$ & VB \\
\hline \multirow{4}{*}{ Innovating (P4) } & $\mathrm{C} 1$ & MB & MB & G & B \\
\hline & $\mathrm{C} 2$ & MB & B & MB & MB \\
\hline & $\mathrm{C} 3$ & $\mathrm{~B}$ & MB & B & B \\
\hline & $\mathrm{C} 4$ & VB & B & B & MB \\
\hline
\end{tabular}

To evaluate the behavior of the model in this hypothetical example, evaluations are provided for four SMEHs, each representing a different maturity level, according to the evaluation performed by the DM and as shown in Table 6 .
Table 5 Qualitative characterization of each SMEH by four DMs considering four criteria

\begin{tabular}{|c|c|c|c|c|c|}
\hline & Criterion & DM1 & DM2 & DM3 & DM4 \\
\hline \multirow{4}{*}{ SMEH 1 } & $\mathrm{C} 1$ & G & MB & MB & G \\
\cline { 2 - 6 } & $\mathrm{C} 2$ & $\mathrm{~B}$ & MB & G & MB \\
\cline { 2 - 6 } & $\mathrm{C} 3$ & G & G & MB & G \\
\cline { 2 - 6 } & $\mathrm{C} 4$ & B & G & G & B \\
\hline
\end{tabular}

\begin{tabular}{|c|c|c|c|c|c|}
\hline & Criterion & DM1 & DM2 & DM3 & DM4 \\
\hline \multirow{4}{*}{ SMEH 2 } & $\mathrm{C} 1$ & VG & VG & G & MB \\
\cline { 2 - 6 } & $\mathrm{C} 2$ & $\mathrm{G}$ & $\mathrm{G}$ & $\mathrm{MB}$ & $\mathrm{MB}$ \\
\cline { 2 - 6 } & $\mathrm{C} 3$ & $\mathrm{G}$ & $\mathrm{VG}$ & $\mathrm{VG}$ & $\mathrm{VG}$ \\
\cline { 2 - 6 } & $\mathrm{C} 4$ & $\mathrm{VG}$ & $\mathrm{G}$ & $\mathrm{VG}$ & $\mathrm{MB}$ \\
\hline
\end{tabular}

\begin{tabular}{|c|c|c|c|c|c|}
\hline & Criterion & DM1 & DM2 & DM3 & DM4 \\
\hline \multirow{4}{*}{ SMEH 3 } & C1 & EG & EG & VG & VG \\
\cline { 2 - 6 } & $\mathrm{C} 2$ & VG & EG & EG & G \\
\cline { 2 - 6 } & $\mathrm{C} 3$ & VG & VG & EG & VG \\
\cline { 2 - 6 } & $\mathrm{C} 4$ & EG & G & EG & EG \\
\hline
\end{tabular}

\begin{tabular}{|c|c|c|c|c|c|}
\hline & Criterion & DM1 & DM2 & DM3 & DM4 \\
\hline \multirow{4}{*}{ SMEH 4 } & $\mathrm{C} 1$ & VB & MB & B & VB \\
\cline { 2 - 6 } & $\mathrm{C} 2$ & VB & B & B & VB \\
\cline { 2 - 6 } & $\mathrm{C} 3$ & B & VB & VB & B \\
\cline { 2 - 6 } & $\mathrm{C} 4$ & B & VB & B & MB \\
\hline
\end{tabular}

Unlike the Fuzzy ELECTRE model, the proposed model will carry out the evaluation in terms of the concordance and dominance that an SMEH has over each of the maturity levels defined above. Likewise, the model incorporates as a variant both, the importance of the DM in the evaluation and the importance of each of the criteria that make up the dimensions. This makes the model flexible for this type of evaluation.

\subsection{Fuzzy ELECTRE Model}

ELECTRE (elimination and (et) choice translating algorithm) is a family of multicriteria evaluation methods, originally developed in 1966 [49], that are part of the methods of over classification or outranking methods. The evolution of ELECTRE methods is described in [50]. One of the fundamental objectives of these methods is to select an alternative by assessing the concordance with a set of desirable points of reference and its discrepancy with respect to other reference points. Generally, these types of models include three ba- 
sic concepts: index of agreement, index of discordance and values or reference thresholds $[12,19,48]$.

According to the criteria that evaluate the level of maturity of a SMEH with respect to big data, a multicriteria evaluation method is proposed, inspired by the structure of a Fuzzy ELECTRE method. The proposed method incorporates for the evaluation the importance of dimensions and decision makers. This method applies the following steps:

Step 1: Definition of the qualitative decision matrix. The proposed model considers a matrix composed of $j$ decision makers (DMs) $(j=1,2, \ldots, n d m)$ and $i$ evaluation criteria $(i=1,2, \ldots, n c)$, which conform a matrix that shows the importance that each DM assigns to each criterion by considering the linguistic values defined in Table 2.

Table 6 Qualitative assessment of the big data maturity level in SMEHs

\begin{tabular}{|c|c|c|c|l|c|}
\hline & Criterion & $\mathrm{DM} 1$ & $\mathrm{DM} 2$ & $\cdots$ & $\mathrm{DMndm}$ \\
\hline \multirow{4}{*}{ SMEHs } & $\mathrm{C} 1$ & $C_{1,1}$ & $C_{1,2}$ & $\cdots$ & $C_{1, n d m}$ \\
\cline { 2 - 6 } & $\mathrm{C} 2$ & $C_{2,1}$ & $C_{2,2}$ & $\cdots$ & $C_{2, n d m}$ \\
\cline { 2 - 6 } & $\vdots$ & $\vdots$ & $\vdots$ & $\ddots$ & $\vdots$ \\
\cline { 2 - 6 } & $\mathrm{Cnc}$ & $C_{n c, 1}$ & $C_{n c, 2}$ & $\cdots$ & $C_{n c, n d m}$ \\
\hline
\end{tabular}

It is worthwhile mentioning that each input of the matrix is described by three values that numerically determine a fuzzy set, as described in Table 3.

$C_{i, j}=\left\{C_{l, i, j}, C_{m, i, j}, C_{u, i, j}\right\}$

Accordingly, the calculation for the matrix is as follows:

$x_{k, i, j}=\frac{\sum_{j=1}^{n d m} i d m_{j} * C_{k, i, j}}{n d m}$

where $k$ indicates the number of maturity levels used for the evaluation $(k=1,2, \ldots, n p) ; i d m_{j}$ indicates the importance of each DM for the evaluation.

The importance of each criterion for a SMEH depends on the mission and vision of the organization. Unlike the traditional Fuzzy ELECTRE model, this model allows to incorporate the importance of each dimension in terms of the mission and vision $\left(i d_{i}\right)$, and in general the business objectives of a SMEH. This way, the estimation of the concordance indices can be carried out as follows:

$i d m_{n, i}=\frac{i d m_{i}}{\sum_{i=1}^{n} c i d m_{i}}$

Due to the structure of each fuzzy set, the structure of the decision matrix can be expressed as:

$x_{k, i}=\frac{\sum_{z=1}^{3} x_{z, k, i}}{3}$ where $z$ indicates the number of components in a fuzzy set ( $z=1$ (low), 2 (medium), 3 (high)).

Finally, the decision matrix can be expressed numerically as follows:

$X=\left[\begin{array}{cccc}x_{11} & x_{12} & \ldots & x_{1 n c} \\ x_{21} & x_{22} & \ldots & x_{2 n c} \\ \vdots & \vdots & \ddots & \vdots \\ x_{n p 1} & x_{n p 2} & \ldots & x_{n p n c}\end{array}\right]$

Step 2: Construction of the normalized decision matrix. The normalization of the matrix is necessary to have the entries of each of the $X$ in the same order of magnitude. The normalized decision matrix is derived as:

$r_{i j}=\frac{1 / x_{i, k}}{\sqrt{\sum_{i=1}^{n c} 1 / x_{i, k}^{2}}}$ (for minimization)

$r_{i j}=\frac{x_{i, k}}{\sqrt{\sum_{i=1}^{n c} x_{i, k}^{2}}}$ (for maximization)

$R=\left[\begin{array}{cccc}r_{11} & r_{12} & \ldots & r_{1 n c} \\ r_{21} & r_{22} & \ldots & r_{2 n c} \\ \vdots & \vdots & \ddots & \vdots \\ r_{n l 1} & r_{n l 2} & \ldots & r_{n l n c}\end{array}\right]$

Step 3: Calculation of the normalized fuzzy decision matrix regarding the criteria. Generally, the fuzzy importance of the criteria can be described by each criterion $\widetilde{w}_{i}=\left(l_{i}, m_{i}, u_{i}\right)$, taking into account the matrices $\widetilde{W_{F}}$ (in Dnashvar, [30]). According to the set of matrices $\widetilde{W}_{F}$, the following procedure can be applied for each criterion:

$\widetilde{G}_{i}=\left(l_{i}, m_{i}, u_{i}\right)$

$l_{i}=\left(l_{i, 1} \otimes l_{i, 2} \otimes \cdots \otimes l_{i, n p}\right)^{1 / n p}$

$m_{i}=\left(m_{i, 1} \otimes m_{i, 2} \otimes \cdots \otimes m_{i, n p}\right)^{1 / n p}$

$u_{i}=\left(u_{i, 1} \otimes u_{i, 2} \otimes \cdots \otimes u_{i, n p}\right)^{1 / n p}$

To locate each criterion in the same order of magnitude, we proceed to the fuzzy normalization $(\mathrm{FN})$ of each criterion:

$\widetilde{w_{F N}}=\left[w_{l, i}, w_{m, i}, w_{u, i}\right]$

where:

$\widetilde{G_{T}}=\left(\sum_{i=1}^{n c} l_{i}, \sum_{i=1}^{n c} m_{i}, \sum_{i=1}^{n c} u_{i}\right)$

$\widetilde{w}_{i}=\left(\frac{l_{i}}{\sum_{i=1}^{n c} l_{i}}, \frac{m_{i}}{\sum_{i=1}^{n c} m_{i}}, \frac{u_{i}}{\sum_{i=1}^{n c} u_{i}}\right)$ 
Step 4: Estimation of the hierarchization matrices. To obtain the hierarchical matrices:

$$
\widetilde{V}=\left[V_{l}, V_{m}, V_{u}\right]
$$

where the entries of each of the matrices can be estimated:

$$
\begin{aligned}
& \widetilde{v_{l, k, i}}=r_{k, i} \times \widetilde{w_{l, i}} \\
& \widetilde{v_{m, k, i}}=r_{k, i} \times \widetilde{w_{m, i}} \\
& \widetilde{v_{l, k, i}}=r_{k, i} \times \widetilde{w_{u, i}}
\end{aligned}
$$

\subsection{Materials and Methods}

To evaluate the behavior of the model with respect to the assessment of the big data maturity level of a SMEH, the following metrics will be used:

- Indices of concordance: The concordance indices $C_{a 1, a 2}^{z}$ represent the degree of dominance that a level of maturity of a SMEH has with regard to each of the maturity levels that have been established as a reference for this study. Thus, the concordance indices can be calculated:

$C_{a 1, a 2}^{z}=\frac{\sum_{i=1}^{n c}\left(w_{z, i, a 1}+w_{z, i, a 2}\right) \cdot i c_{i}}{n c c}$

where $n c c$ is the number of criteria that meet the dominance value for each of the matching matrices; and $i c_{i}$ is the index of importance of each criterion $i$.

The equations above show a conditional sum in terms of the criteria in which the proposal $a_{1}$ (base SMEH) dominates proposal $a_{2}$, where $n c c$ indicates the number of columns that fulfill the condition.

- Indices of Discrepancy or mismatch:These indices show the discrepancy between a proposal $a_{1}$ (base SMEH) and a proposal $a_{2}$ (reference maturity levels). These discrepancy indices are defined as follows:

$D_{a_{1}, a_{2}}^{z}=\frac{\sum_{i^{*}=1}^{n d}\left|v_{a_{1}, i^{*}}^{z}-v_{a_{2}, i^{*}}^{z}\right|}{\sum_{i=1}^{n d}\left|v_{a_{1}, i}^{z}-v_{a_{2}, i}^{z}\right|}$

where, $z=3$.

- Maturity level: The dominance of the maturity level of an SMEH over the reference maturity levels, that have been established for this study, is stronger when the levels of agreement $C_{a_{1}, a_{2}}^{z}$ are high, whereas the discrepancy levels $D_{a_{1}, a_{2}}^{z}$ are low, and additionally the following condition will be met:

$C_{a 1, a 2}^{z} \geq \bar{C} ; \quad D_{a 1, a 2}^{z} \geq \bar{D}$

where $\bar{C}$ is the average of the concordance values $C_{a_{1}, a_{2}}^{z}$, and $\bar{D}$ represents the average of the discrepancy values $D_{a 1, a 2}^{z}$.

\section{Experimental Results}

To illustrate the behavior of the proposed model, a hypothetical SMEH with the following qualitative evaluation characteristics is considered for this study:

Table 7 Qualitative evaluation of a reference SMEH

\begin{tabular}{|c|c|c|c|c|c|}
\hline & Criterion & DM1 & DM2 & DM3 & DM4 \\
\hline \multirow{4}{*}{ SMEH (P0) } & $\mathrm{C} 1$ & $\mathrm{G}$ & VG & VG & G \\
\cline { 2 - 6 } & $\mathrm{C} 2$ & MB & VG & MB & MB \\
\cline { 2 - 6 } & $\mathrm{C} 3$ & MB & G & MB & MB \\
\cline { 2 - 6 } & $\mathrm{C} 4$ & G & VG & MB & MB \\
\hline
\end{tabular}

Step 1: Taking into account Table 4, the numerical decision matrix that is derived from the qualitative decision matrix, in terms of the fuzzy sets representing each quality, is

$$
X=\left[\begin{array}{llll}
6.0 & 4.0 & 3.5 & 4.5 \\
6.0 & 4.5 & 5.5 & 4.0 \\
5.0 & 3.5 & 2.5 & 3.3 \\
4.0 & 2.5 & 3.3 & 2.3 \\
3.3 & 2.8 & 2.3 & 2.0 \\
1.8 & 2.3 & 1.5 & 2.3
\end{array}\right]
$$

Step 1: From this numerical decision matrix the normalized numerical decision matrix is obtained:

$$
R=\left[\begin{array}{llll}
0.230 & 0.205 & 0.189 & 0.246 \\
0.230 & 0.230 & 0.297 & 0.219 \\
0.192 & 0.179 & 0.135 & 0.178 \\
0.153 & 0.128 & 0.175 & 0.123 \\
0.125 & 0.141 & 0.121 & 0.109 \\
0.067 & 0.115 & 0.081 & 0.123
\end{array}\right]
$$

Step 3: Subsequently, the process continues to obtain the fuzzy sets that determine each of the criteria. Matrix $\widetilde{W}$ represents the fuzzy sets obtained for each of the evaluation criteria.

$$
\widetilde{W}=\left[\begin{array}{lll}
0.323 & 0.323 & 0.323 \\
0.240 & 0.242 & 0.243 \\
0.215 & 0.214 & 0.214 \\
0.220 & 0.219 & 0.219
\end{array}\right]
$$


Step 3: According to matrices $R$ and $\widetilde{W}$, we proceed to obtain the hierarchical matrices that allow the estimation of the concordance indices $C_{a_{1}, a_{2}}^{z}$, and also of the indices of discordance, $D_{a_{1}, a_{2}}^{z}$.

$V 1=\left[\begin{array}{lllll}0.0746 & 0.0493 & 0.0407 & 0.0543 \\ 0.0746 & 0.0555 & 0.0639 & 0.0483 \\ 0.0622 & 0.0432 & 0.0290 & 0.0392 \\ 0.0497 & 0.0308 & 0.0378 & 0.0271 \\ 0.0404 & 0.0339 & 0.0261 & 0.0241 \\ 0.0217 & 0.0277 & 0.0174 & 0.0271\end{array}\right]$

$V 2=\left[\begin{array}{llll}0.0745 & 0.0497 & 0.0405 & 0.054 \\ 0.0745 & 0.0559 & 0.0637 & 0.0481 \\ 0.0621 & 0.0435 & 0.0289 & 0.0391 \\ 0.0497 & 0.0310 & 0.0376 & 0.0270 \\ 0.0404 & 0.0342 & 0.0260 & 0.0240 \\ 0.0217 & 0.0279 & 0.0173 & 0.0270\end{array}\right]$

$V 3=\left[\begin{array}{llll}0.0745 & 0.0500 & 0.0405 & 0.0540 \\ 0.0745 & 0.0562 & 0.0637 & 0.0480 \\ 0.0621 & 0.0437 & 0.0289 & 0.0390 \\ 0.0496 & 0.0312 & 0.0376 & 0.0270 \\ 0.0403 & 0.0343 & 0.0260 & 0.0240 \\ 0.0217 & 0.0281 & 0.0173 & 0.0270\end{array}\right]$

- Based on the hierarchical matrices, the concordance indices obtained are:

Table 8 Indices of concordance for each hierarchization matrix

\begin{tabular}{|l|c|l|l|l|l|}
\hline$C_{1}$ & & $C_{2}$ & & $C_{3}$ & \\
\hline$C_{0,1}^{1}$ & 8.2 & $C_{0,1}^{2}$ & 10.25 & $C_{0,1}^{3}$ & 12.3 \\
\hline$C_{0,2}^{1}$ & 6.45 & $C_{0,2}^{2}$ & 8.0625 & $C_{0,2}^{3}$ & 9.675 \\
\hline$C_{0,3}^{1}$ & 6.0125 & $C_{0,3}^{2}$ & 7.5 & $C_{0,3}^{3}$ & 8.9875 \\
\hline$C_{0,4}^{1}$ & 5.6625 & $C_{0,4}^{2}$ & 7.0625 & $C_{0,4}^{3}$ & 8.4625 \\
\hline$C_{0,5}^{1}$ & 5.225 & $C_{0,5}^{2}$ & 6.4375 & $C_{0,5}^{3}$ & 7.65 \\
\hline
\end{tabular}

- The average values of the concordance and discrepancy indices, obtained from the hierarchical matrices are the following: The hierarchization of the indices of concordance and discrepancy can be observed in the previous
Table 9 Indices of concordance and dominance for the considered SMEH

\begin{tabular}{|c|c|c|c|c|c|}
\hline \multicolumn{3}{|c|}{ Concordance } & \multicolumn{3}{c|}{ Discrepancy } \\
\hline$C_{0,1}$ & 10.250 & 1 & $D_{0,1}$ & 0.833 & 1 \\
\hline$C_{0,2}$ & 8.063 & 2 & $D_{0,2}$ & 0.000 & 2 \\
\hline$C_{0,3}$ & 7.500 & 3 & $D_{0,3}$ & 0.000 & 2 \\
\hline$C_{0,4}$ & 7.063 & 4 & $D_{0,4}$ & 0.000 & 2 \\
\hline$C_{0,5}$ & 6.438 & 5 & $D_{0,5}$ & 0.000 & 2 \\
\hline Average & 7.863 & & Average & 0.167 & \\
\hline
\end{tabular}

table. This allows to describe the dominance of the level of maturity of the modeled SMEH, based on the value of maturity defined by the DM for each level. It can be highlighted that the highest concordance value occurred with respect to the level of maturity which corresponds to the level of "innovating", where the level of discrepancy also was the highest, which reinforces even more that the modeled SMEH should be considered as "innovating" in terms of its capability in big data management. An overview of the maturity levels obtained by each of the SMEHs, that were evaluated qualitatively, is shown in Table 5. The SMEHs can be mapped on the linguistic variable that shows the fuzzy sets associated with each level of maturity (see Figure 2 underneath). In this figure, it can be observed that $S M E H_{1}$ achieved a level of maturity between "integrating" and "innovating", where the level of membership for "integrating" was $u_{i}=0.91$, while the level for "innovating" was $u_{i}=$ 0.17 . This indicates that this SMEH is at the level of "integrating", whereas $\mathrm{SMEH}_{4}$ was placed at a "inexistent" level with a membership value of $u_{i}=1.0$. Finally, $\mathrm{SMEH}_{2}$ and $\mathrm{SMEH}_{3}$ were located at higher membership levels, i.e. "innovating", with a membership value of $u_{i}=1.0$, which is in accordance with the characteristics evaluated for each SMEH.

To observe the evolution of maturity levels of a SMEH, the profile of maturity limits is defined for each criterion $i$ by the following vector:

$b_{i}=\left(b_{i, 1}, b_{i, 2}, \ldots, b_{i, n p}\right)$

Each of the maturity levels for a criterion is defined by the group of DMs that were considered for this study. These levels show how DMs perceive maturity in terms of the criteria that define an evaluation dimension, and agree with the fuzzy sets shown in Figure 2.

In Figure 3 the evolution of the level of maturity for $\mathrm{SMEH}_{4}$ can be observed for each criterion, where each criterion undergoes a qualitative progressive improvement in the evaluation of each of the dimensions of maturity. This figure 


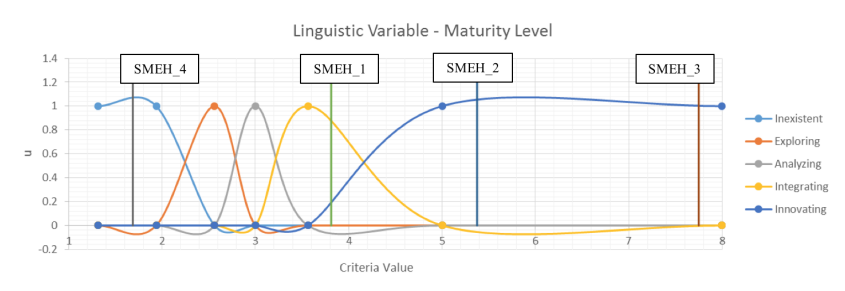

Fig. 2 Mapping for the different levels of maturity, obtained for the SMEHs considered in Table 5, regarding the linguistic variable that represents the maturity level

shows the initial as well as the final profiles of criteria. The initial profile shows the intrinsic importance of each criterion for $\mathrm{SMEH}_{4}$. However, to achieve a homogeneous maturity level for each criterion over time, it is necessary that the importance associated with criteria $\left(i c_{i}\right)$, which have a slower evolution, has a greater importance index value. This will be reflected directly in a greater amount of resources and in a continuous improvement, in particular of the criteria related to the dimensions "processes" and "technology", which contribute in defining big data maturity. This improvement should be observed for the most relevant aspects that describe each criterion, as defined above.

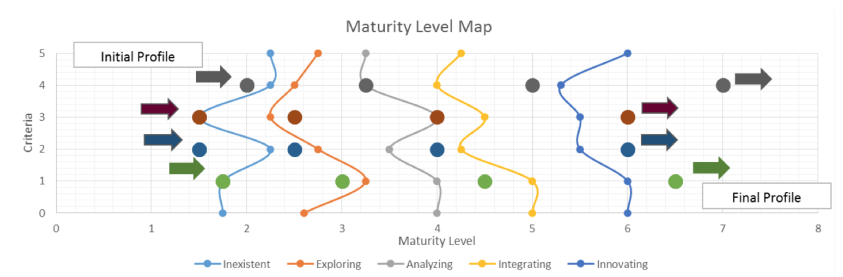

Fig. 3 Mapping of the evolution of each criterion for the assessment of $\mathrm{SMEH}_{4}$

\section{Conclusions and future work}

This paper presents a fuzzy model, based on the structure of a Fuzzy ELECTRE model, for a multicriteria evaluation of big data maturity in SMEHs. The model incorporates the importance of each evaluation criterion, as well as the importance of each DM with respect to the final evaluation. The proposed model also helps to diagnose, how prepared an organization is for introducing big data initiatives or technologies (big data readiness). The main contribution of this research consists in applying the Fuzzy type of ELECTRE in a new context, measuring the level of big data maturity in SME in the healthcare sector (SMEHs). Unlike traditional ELECTRE models, the proposed model was modified, considering the dominance of a proposed maturity evaluation for a SMEH over a series of proposals that show the level of maturity of a SMEH and taking into account the defined dimensions that are similar to the ones used by the TDWI methodology.

The proposed model allowed a more comprehensive evaluation of big data maturity of a SMEH as traditional models. This is because a linguistic variable is used that shows the qualitative characteristics that define a level of maturity and also as a map of characteristics that define a big data maturity profile for each of the dimensions. That allows to identify the intrinsic importance of each dimension in a SMEH and to modify the importance that should be given to each criterion or dimension with the aim to achieve a homogeneous evolution of maturity over time.

With respect to future work, a methodology could be engineered that automatically establishes the level of importance for each criterion to achieve a homogeneous evolution of maturity, in terms of the importance that the model allows to assign to each of the participating DMs. This importance could be assigned in terms of fuzzy sets with the aim to create multidimensional spatial models, based on the effect of the Cartesian product between the importance of DMs and each assessed criterion.

\section{Compliance with Ethical Standards}

Conflict of Interest: The authors declare that they have no conflict of interest.

Ethical approval: This article does not contain any studies with human participants or animals performed by any of the authors.

Informed consent: This was not necessary because no individual participants were involved.

\section{References}

1. Qinghua Lu, Zheng Li, Weishan Zhang, Laurence T. Yang (2017) Autonomic deployment decision making for big data analytics applications in the cloud, Soft Computing 21 (16), 4501-4512.

2. Diego J. Bodas-Sagi, José M. Labeaga Big (2018) Data and Health Economics: Opportunities, Challenges and Risks. International Journal of Interactive Multimedia and Artificial Intelligence 4 (7), $47-52$.

3. Bernard Marr (2015) How Big Data Is Changing Healthcare, Forbes. Available at https:// www . forbes. com/sites/bernardmarr/2015/04/ 21/how-big-data-is-changing-healthcare/ \#39b365dd2873.

4. Donna M. Schaeffer, Patrick C. Olson, (2014) Big Data Options For Small And Medium Enterprises, Review of Business Information Systems 18 (1): 41-46.

5. Rogelio Víez (2012) Alta Gerencia: Horarios flexibles en el trabajo motivan a los empleados y aumentan la productividad. Available at https://www.larepublica.co/alta-gerencia and https://tinyurl. com/y8ra7koj. 
6. Hee Dae Kim, In Lee, Choong Kwon Lee (2011) Building Web 2.0 enterprises: A study of small and medium enterprises in the United States, International Small Business Journal 31(2): 156174.

7. Luis Hernando Palacio Palacio (2015) Cálculo de los Parámetros de la Distribución de Weibull. Mantenimiento en Latinoamérica 7 (1), 42-44. Available at http://mantenimientoenlatinoamerica.com/pdf/ ML $\backslash \% 20$ Volumen $\backslash \% 207-1$.pdf.

8. Arturo González-Ferrer, Germán Seara, Joan Cháfer, Julio Mayol (2018) Generating Big Data Sets from Knowledge-based Decision Support Systems to Pursue Value-based Healthcare, International Journal of Interactive Multimedia and Artificial Intelligence 4 (7), $42-46$

9. GS1 Ireland (2017). Healthcare SMEs Lead the Way with GS1 Standards. Available at https://www. gs1ie.org/Healthcare/Resources/Case-Studies/ Healthcare-SMEs-Lead-the-Way-with-GS1-Standards . html.

10. Mateo Santos (2014). Las Pymes ya Están Usando Big Data e Inteligencia De Datos. Available at http://www.enter.co/ especiales/enterprise/big-data-tecnologia-pymes/

11. Vahid Garousi, Michael Felderer, Tuna Hacaloglu, (2017) Software test maturity assessment and test process improvement: A multivocal literature review, Information and Software Technology $85: 16-42$

12. Babak Daneshvar Rouyendegh, Serpil Erol (2012) Selecting the Best Project Using the Fuzzy ELECTRE Method, Mathematical Problems in Engineering 2012: 12 pages.

13. F. J Cabrerizo, R. Al-Hmouz, A. Morfeq, A. S. Balamash, M. A. Martínez, E. Herrera-Viedma (2017) Soft consensus measures in group decision making using unbalanced fuzzy linguistic information, Soft Computing 21 (11), 3037-3050 .

14. Luis G. Perez, Francisco Mata, Francisco Chiclana, Gang Kou, Enrique Herrera-Viedma (2016) Modelling influence in group decision making, Soft Computing 20 (4), 1653-1665

15. Nicola Capuano, Francisco Chiclana, Hamido Fujita, Enrique Herrera-Viedma, Vincenzo Loia (2018) Fuzzy group decision making with incomplete information guided by social influence. IEEE Transactions on Fuzzy System 26 (3) 1704-1718.

16. Jian Wu, Ruoyun Xiong, Francisco Chiclana (2016) Uninorm trust propagation and aggregation methods for group decision making in social network with four tuples information, Knowledge-Based Systems 96, 29-39.

17. Juan Pablo Anún, Rodrigo Alarcón (2014) Ranking Projects of Logistics Platforms: A Methodology Based on the Electre Multicriteria Approach, Procedia - Social and Behavioral Sciences 160: 5-14.

18. Benjamin Dehe, David Bamford (2015) Development, test and comparison of two Multiple Criteria Decision Analysis (MCDA) models: A case of healthcare infrastructure location, Expert Systems with Applications 42 (19): 6717-6727.

19. Silvia Angilella, Sebastiano Mazzu (2015) The financing of innovative SMEs: A multicriteria credit rating model, European Journal of Operational Research 244 (2): 540-554.

20. Jiunn-Woei Lian, Chih-Kun Ke(2016) Using a modified ELECTRE method for an agricultural product recommendation service on a mobile device, Computers \& Electrical Engineering 56: 277288.

21. Parth Chandarana, M. Vijayalakshmi (2014) Big Data analytics frameworks, Proceedings of the 2014 International Conference on Circuits, Systems, Communication and Information Technology Applications (CSCITA), Mumbai, pp. 430-434.

22. María Díaz-Ley, Félix García, Mario Piattini (2010) MIS-PyME software measurement capability maturity model - Supporting the definition of software measurement programs and capability determination, Advances in Engineering Software 41 (10-11): 12231237.
23. Javier Garzás, Francisco J. Pino, Mario Piattini, Carlos Manuel Fernández (2013) A maturity model for the Spanish software industry based on ISO standards, Computer Standards \& Interfaces 35 (6), 616-628.

24. Yilmaz Goksen, Eda Cevik, Huseyin Avunduk (2015) A Case Analysis on the Focus on the Maturity Models and Information Technologies, Procedia Economics and Finance 19, 208-216.

25. Marco Kuhrmann, Thomas Ternité, Jan Friedrich, Andreas Rausch, Manfred Broy (2016) Flexible software process lines in practice: A metamodel-based approach to effectively construct and manage families of software process models, Journal of Systems and Software 121, 49-71.

26. Yoshitaka Kuwata, Kentaro Takeda, Hiroshi Miura (2014) A Study on Maturity Model of Open Source Software Community to Estimate the Quality of Products, Procedia Computer Science 35, 1711-1717.

27. Maximilian Röglinger, Jens Pöppelbuß, Jörg Becker, (2012) "Maturity models in business process management", Business Process Management Journal 18 (2), 328-346.

28. Jasmien Lismont, Jan Vanthienen, Bart Baesens, Wilfried Lemahieu(2017) Defining analytics maturity indicators: A survey approach, International Journal of Information Management 37 (3), 114-124

29. Mihály Görög (2016) A broader approach to organisational project management maturity assessment, International Journal of Project Management 34 (8), 1658-1669.

30. Diogo Proença, José Borbinha (2016) Maturity Models for Information Systems - A State of the Art, Procedia Computer Science $100,1042-1049$.

31. Henrik von Scheel, Gabriella von Rosing, Krzysztof Skurzak, Maria Hove (2015) BPM and Maturity Models, In Editor(s): Mark von Rosing, August-Wilhelm Scheer, Henrik von Scheel, The Complete Business Process Handbook, Morgan Kaufmann, 399430.

32. Ayca Tarhan, Oktay Turetken, Hajo A. Reijers (2016) Business process maturity models: A systematic literature review, Information and Software Technology 75, 122-134.

33. Fern Halper, David Stoler (2014) TDWI Analytics Maturity Model Guide Transforming Data with Intelligence. White Paper, available at https://tdwi.org/whitepapers/2014/10/ tdwi-analytics-maturity-model-guide.aspx.

34. Jorge D. Camba, Manuel Contero, Pedro Company (2016) Parametric CAD modeling: An analysis of strategies for design reusability, Computer-Aided Design 74, 18-31.

35. Info-Tech Research group (2017) Big data maturity assessment tool. Available at https://www.infotech.com/ research/ss/leverage-big-data-by-starting-small/ it-big-data-maturity-assessment-tool.

36. Carlos A. Bana e Costa (Ed.) Readings in Multiple Criteria Decision Aid, Springer-Verlag, Berlin, Heidelberg, 1990.

37. Eric Jacquet-Lagrèze (1995) An Application of the UTA Discriminant Model for the Evaluation of R \& D Projects. In: Pardalos P.M., Siskos Y., Zopounidis C. (eds) Advances in Multicriteria Analysis. Nonconvex Optimization and Its Applications, vol 5. Springer, Boston, MA

38. Eric Jacquet-Lagrèze, J. Siskos (1982) Assessing a set of additive utility functions for multicriteria decision-making, the UTA method, European Journal of Operational Research 10 (2), 151164.

39. Vincent Mousseau, José Rui Figueira, Jean-Philippe Naux (2001) Using assignment examples to infer weights for ELECTRE TRI method: Some experimental results, European Journal of Operational Research 130 (2), 263-275.

40. Yucheng Dong, Wenqi Liu, Francisco Chiclana, Enrique HerreraViedma, Francisco Javier Cabrerizo (2017) Group decisionmaking based on heterogeneous preference relations with selfconfidence, Fuzzy Optimization and Decision Making 16 (4) 429_ 447. 
41. Yucheng Dong, Cong-Cong Li, Francisco Chiclana, Enrique Herrera-Viedma (2016) Average-case consistency measurement and analysis of interval-valued reciprocal preference relations, Knowledge-Based Systems 114, 108-117.

42. Hengjie Zhang, Yucheng Dong, Enrique Herrera-Viedma (2018) Consensus building for the heterogeneous large-scale GDM with the individual concerns and satisfactions, IEEE Transactions on Fuzzy System 26(2), 884-898.

43. Yujia Liu, Changyong Liang, Francisco Chiclana, Jian Wu (2017) A trust induced recommendation mechanism for reaching consensus in group decision making, Knowledge-Based Systems Volume $119,221-231$.

44. Denis Bouyssou, Thierry Marchant (2007) An axiomatic approach to noncompensatory sorting methods in MCDM, I: The case of two categories, European Journal of Operational Research 178 (1), 217-245.

45. Ralph L. Keeney, Howard Raiffa, Decisions with Multiple Objectives: Preferences and Value Tradeoffs. Cambridge University Press, Cambridge \& New York, 1993.

46. José Figueira, Bernard Roy (2002) Determining the weights of criteria in the ELECTRE type methods with a revised Simos' procedure, European Journal of Operational Research 139 (2), 317-326.

47. Jean-Pierre Brans, Yves De Smet (2016) PROMETHEE Methods. In: Greco S., Ehrgott M., Figueira J. (eds) Multiple Criteria Decision Analysis. International Series in Operations Research \& Management Science, vol 233. Springer, New York, NY, 187-219.

48. Mehmet Sevkli (2009) An application of the fuzzy ELECTRE method for supplier selection, International Journal of Production Research, 48 (12), 3393-3405.

49. R. Benayoun, B. Roy, B. Sussmann (1966) ELECTRE : Une míhode pour guider le choix en présence de points de vue multiples, Note de travail $\mathrm{n}^{\circ} 49$ de la Direction Scientifique de la SEMA.

50. José Rui Figueira, Vincent Mousseau, Bernard Roy,(2016) ELECTRE Methods. In: Greco S., Ehrgott M., Figueira J. (eds) Multiple Criteria Decision Analysis. International Series in Operations Research \& Management Science, vol 233. Springer, New York, NY pp. $155-182$. 